\title{
Adiponectin and hemoglobin levels in overweight and obese pregnant mothers in early pregnancy
}

\begin{abstract}
Introduction: Body mass index (BMI) has constantly been associated with adverse health outcomes and, to a certain extent, adverse pregnancy outcome. The combination of increased maternal adiposity and reduction in insulin sensitivity influenced by hormonal products appears to be the causative factors in insulin resistance during gestational period. Adiponectin, which is one of the adipocyte-secreted hormones, was reported to possess insulin-sensitizing properties and appears to play a role in this metabolic syndrome. Accumulating literatures also suggested that high maternal hemoglobin level has an association with increased in incidence of adverse pregnancy outcomes which included the occurrence of gestational diabetes mellitus.Objective: The main objective of our study is to evaluate the relationship between patients' BMI, which is the risk factor for most metabolic disorders, with plasma adiponectin and hemoglobin levels among the antenatal subjects in the local population.Methods: This is a cross-sectional study involving 104 pregnant mothers that were recruited from the Bangi, Kajang and Seri Kembangan polyclinics, sosiodemografic and antenatal data were collected through a simple questionnaire after informed consent taken. All patients were in their first trimester of pregnancy. Patients were subcategorized according to their BMI into 4 categories: underweight, normal weight, overweight and obese. A volume of $3 \mathrm{ml}$ peripheral blood aspiration was taken by trained phlebotomist. Blood plasma was separated and stored at $-80 \hat{\mathrm{A}}^{\circ} \mathrm{C}$ prior to tests. Plasma adiponectin level was measured using commercialized ELISA assay kit. Hemoglobin level was determined using Sysmex KX-21 Hematology Analyzer. Results were analyzed using SPSS version 15.Results: A total of 104 patients enrolled in the study, 12 patients $(11.54 \%)$ were underweight; 57 patients $(54.81 \%)$ were normal weight; 25 patients $(24.04 \%)$ were overweight; and 10 patients $(9.62 \%)$ were obese. A significant difference in hemoglobin level was found between underweight and obese pregnant mothers, as well as between normal weight and obese pregnant mothers. A significant difference in plasma adiponectin level was found between underweight and overweight pregnant mothers, as well as between underweight and obese pregnant mothers. Significant difference in plasma adiponectin level was also found between normal weight and obese pregnant mothers.Conclusion: We concluded that increased early pregnancy BMI, is highly associated with low plasma adiponectin level and increased hemoglobin level in our local population.
\end{abstract}

Keyword: Adiponectin; BMI; Body mass index; Hemoglobin; Obesity; Pregnancy 